Check for updates

Cite this: RSC Adv., 2019, 9, 3092

\title{
Moving mechanisms of the three-phase contact line in a water-decane-silica system
}

\author{
Wenxiu Zheng, Chengzhen Sun, Boyao Wen and Bofeng Bai (iD)*
}

The movement of the three-phase contact line with chain molecules in the liquid phase displays more complex mechanisms compared to those in the usual liquid-liquid-solid systems and even to the gasliquid-solid systems controlled by the traditional single-molecule adsorption-desorption mechanisms. By introducing decane molecules with chain structures, we demonstrate from molecular dynamics insights that the moving mechanism of the contact line in a water-decane-silica system is totally different from traditional mechanisms. Three different wettability-related moving mechanisms including "Roll up", "Piston" and "Shear" are revealed corresponding to the hydrophilic, intermediate and hydrophobic three-phase wettability, respectively. In the "Roll up" mechanism, the decane molecules are rolled up by the competitively adsorbed water molecules and then move forward under the driving force; when the "Piston" mechanism happens, the decane molecules are pushed by the piston-like water phase owing to the comparable adsorption interactions of the two liquids on the solid surface; in the "Shear" mechanism, the contact line is hard to drive due to the stronger decane-silica interactions but the decane molecules far away from the solid surface will move forward. Besides, the time-averaged velocity of the moving contact line is greatly related to the moving mechanisms. For the "Roll up" mechanism, the contact line velocity increases first and then reaches a steady value; for the "Piston" mechanism, the contact line velocity has a maximum value at the start-up stage and then decreases to a stable value; for the "Shear" mechanism, the contact line velocity fluctuates around zero due to the thermal fluctuation of the molecules. Additionally, the mean distance from Molecular Kinetics Theory increases with decreasing hydrophilicity and the displacement frequency in "Roll up" mechanism is 2 orders of magnitude higher than that in the "Piston" mechanism, further demonstrating the different moving mechanisms from a quantitative point of view.

Received 26th November 2018

Accepted 4th January 2019

DOI: $10.1039 / c 8 \mathrm{ra09715a}$

rsc.li/rsc-advances describe the moving contact line. ${ }^{\mathbf{1 6}, 17}$ Although MKT shows good agreement with the experimental data for liquid-liquid displacement, ${ }^{\mathbf{1 8 - 2 0}}$ it is still in doubt how the two liquids displace in the contact line region since the MKT parameters of the two-liquid systems are greatly influenced by the properties of the two liquids and need more physical explanations. Therefore, researchers have tried to establish a link between the one-liquid systems and the two-liquid systems, which could give a clearer understanding about the physical origins of the moving contact line. ${ }^{21-23}$ The source of this problem is the moving mechanism of the contact line understanding the displacing mode of the molecules near the contact line. The moving mechanism of the contact line in a gas-liquid-solid (GLS) system is almost clear since a gas-liquid interface is a lowdensity zone. In this case, the single-molecule adsorptiondesorption mechanism makes the contact line move forward. ${ }^{24}$ Oppositely, for the LLS systems, the contact line zone is dense with a density comparable to those of the two liquids. Some researchers believe that the single-molecule adsorptiondesorption mechanism is still valid and the molecules in the front need to leave empty sites for the latter molecules, and then
State Key Laboratory of Multiphase Flow in Power Engineering, Xi'an Jiaotong University, Xi'an, Shaanxi, 710049, China. E-mail: bfbai@mail.xjtu.edu.cn; Tel: $+86-029-82665316$ 
the latter molecules move forward.$^{23}$ However, some researchers have speculated that the single-molecule adsorption-desorption mechanism may not be competent in the case of LLS systems due to a significantly larger volume of unit flow than that expected from the single-molecule adsorption-desorption mechanism. $^{21,22,25}$

Chain molecules are widely encountered in the oil recovery and chemical industries, etc. Meanwhile, surfactants or polymers are commonly used in many industries, which also have long-chain structures. In a LLS system with chain molecules, the moving contact line is greatly affected by the adsorption properties of the chain molecules on the solid surfaces, ${ }^{26}$ which display orientation arrangement in the adsorption layers. ${ }^{27,28}$ The orientation arrangement is determined by the solid-liquid interactions and the chain structures of the liquid molecule. Researchers showed that the chain molecules tend to be parallel to the solid surfaces ${ }^{27}$ and the chain length has obvious effects on the contact line friction. ${ }^{26,28}$ For this case, the singlemolecule adsorption-desorption mechanism may be invalid since the molecules on the surface are hard to jump forward. Besides, a chain molecule will have torsion during its move, which exerts local deformations at the nanoscale near the contact line; ${ }^{29}$ the local deformations will induce extra unbalanced forces due to the interfacial deformations, thereby affecting the moving contact line. In short, the moving mechanism of the contact line in a LLS system with chain molecules is very crucial for many industries. Until now, this issue has not been examined systematically, especially in the systems with different surface wettabilities.

To better understand the moving mechanism of the threephase contact line, a molecular level investigation on the displacing processes of the two kinds of liquid molecules near the contact line zone is of crucial importance. Molecular dynamics (MD) simulation is an effective method capturing the movements of the atomic particles ${ }^{30,31}$ controlled by Newton's laws of motion. ${ }^{32,33}$ It can obtain useful information about the moving contact line $\mathrm{e}^{34}$ at the molecular level. Therefore, we present a systematical study of the moving contact line in a waterdecane-silica system from molecular insights by using the MD simulations. Different three-phase wettabilities are adopted and the adsorption properties of the liquid molecules are obtained by the statistical method. Several moving mechanisms are shown for the three-phase contact line, which are related to the three-phase wettability.

\section{Simulation model}

We perform the MD simulations in a three-phase system, where the two liquids are placed on a kind of silica surface ( $\alpha$-quartz, $28.4 \mathrm{~nm} \times 6 \mathrm{~nm}$ ), as shown in Fig. 1. The MD simulations are implemented by the Large-scale Atomic/Molecular Massively Parallel Simulator (LAMMPS) software. The molecules are confined in a simulation box through periodic boundary conditions in the directions parallel to the silica surface and reflective boundary conditions in the direction vertical to the silica surface. We employ water and a kind of chain alkane $n$ decane $\left(\mathrm{C}_{10}\right)$ to be the two kinds of liquids. The simulation system contains $1560 \mathrm{C}_{10}$ and $16245 \mathrm{H}_{2} \mathrm{O}$ of transferable intermolecular potential with 3 points (TIP3P) model and the structures of the liquid molecules are shown in Fig. 1. For the silica surface, the non-bridging oxygen atoms which bonded to only one silicon atom are attached to the hydrogen atoms. ${ }^{35}$ In order to better analyze the characteristics of the three-phase contact line, the silica atoms are fixed in the simulations to avoid the weak vibrations of the surface from the collisions with $\mathrm{C}_{10}$ and $\mathrm{H}_{2} \mathrm{O}$ molecules. All simulation cases are initially run for $2 \mathrm{~ns}$ to allow the systems to reach the equilibrium in the canonical (NVT) ensemble. Then a wall $(6 \mathrm{~nm} \times 6.4 \mathrm{~nm})$ is added in the left of the simulation box, as shown in Fig. 1. An external force $F$ to every wall atom is added to drive the system. A simulation run for $5 \mathrm{~ns}$ is firstly presented. As the simulation periods for a system to achieve the steady state change with different adding forces, another $5 \mathrm{~ns}$ run is continued if the system does not reach the steady state. In the simulations, the time step is set to $1.0 \mathrm{fs}$ and the moving process of the threephase contact line is recorded with a period of 10000 timesteps. The system temperature is kept at $300 \mathrm{~K}$ in the NVT ensemble.

To simulate the interactions among the atoms, several potential models are adopted. For the added wall, the adaptive intermolecular reactive empirical bond-order (AIREBO) potential model is applied, whose form and corresponding parameters can be found in the work by Stuart et $a l^{36}$ For the liquid molecules and the silica atoms, Lennard-Jones (L-J) potential model coupling with the coulombic potential is employed. To improve the computational efficiency, only the neighboring particles within a certain cutoff radius $\left(r_{\text {cut }}\right)$ are included in the force calculation, since the distant particles have a negligible contribution. In this work, $r_{\text {cut }}$ is set to $1.0 \mathrm{~nm}$, which is acceptable and widely used by many researchers. ${ }^{32}$ The longrange coulombic interactions are handled with the particleparticle particle-mesh (PPPM) method. To weaken the interactions between the added wall and the molecules near the contact line area, the length of the water side along $x$-direction is set to be long enough $(14 \mathrm{~nm})$. In this case, the van der Waal's interactions and the coulombic interactions (the charges of the added wall atoms are zero) between the wall atoms and the molecules near the contact line can be ignored.

The terms of the Lennard-Jones (L-J) potential (eqn (1)) and the coulombic potential (eqn (2)) can be described as follows:

$$
\phi\left(r_{\mathrm{ij}}\right)=4 \varepsilon\left[\left(\frac{\sigma}{r_{\mathrm{ij}}}\right)^{12}-\left(\frac{\sigma}{r_{\mathrm{ij}}}\right)^{6}\right]
$$

where $r_{\mathrm{ij}}$ is the distance between atoms $\mathrm{i}$ and $\mathrm{j}$, and $\varepsilon$ and $\sigma$ are the energy parameter and the length scale, respectively.

$$
\phi_{\mathrm{c}}\left(r_{\mathrm{ij}}\right)=\frac{C q_{\mathrm{i}} q_{\mathrm{j}}}{\varepsilon_{0} r_{\mathrm{ij}}}
$$

where $q_{\mathrm{i}}$ and $q_{\mathrm{j}}$ are the charges of atoms i and j, $C$ is the energyconversion constant, $\varepsilon_{0}$ is the dielectric constant in vacuo.

The interactions between silica atoms and atoms in $\mathrm{H}_{2} \mathrm{O}$ and $\mathrm{C}_{10}$ molecules are modeled using the hybrid potential model. The L-J potential parameters between the crossing atoms are 

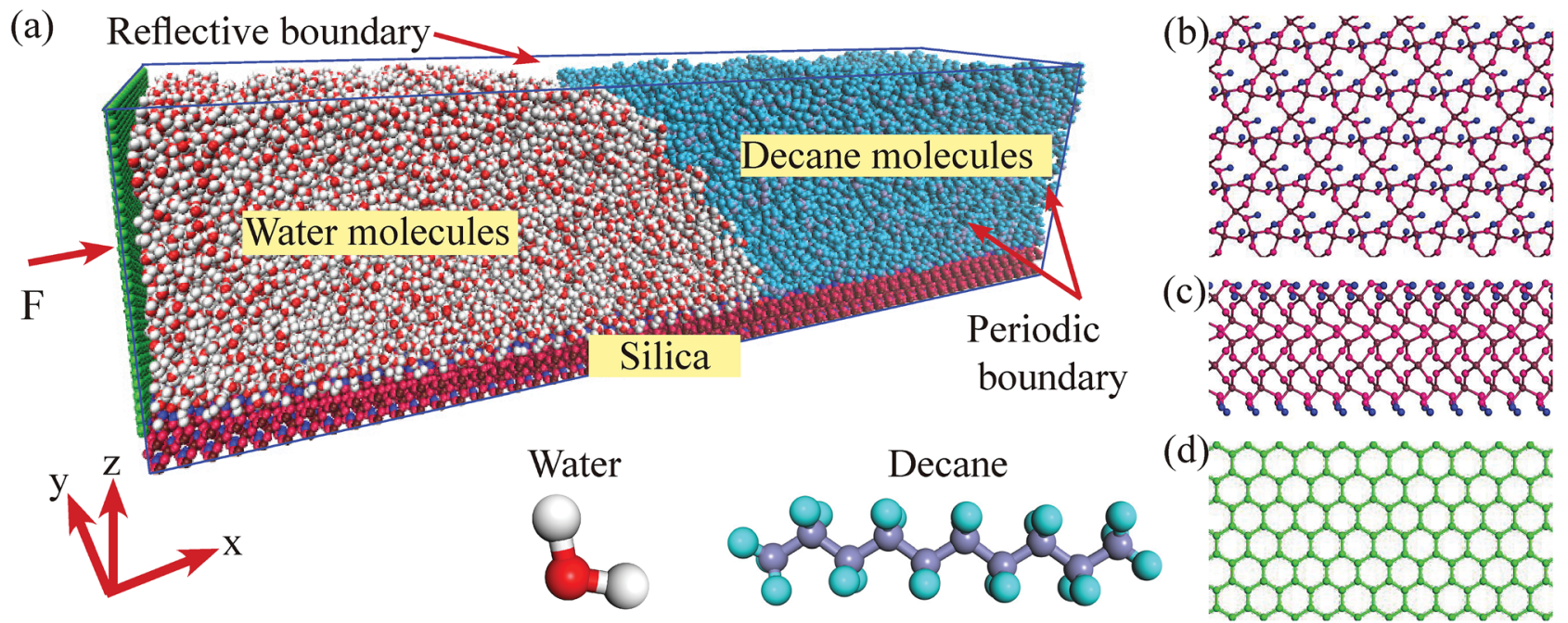

Fig. 1 Molecular dynamics model. (a) Simulation system and the structures of the water and decane molecules. (b) Top view of the silica surface. (c) Side view of the silica surface. (d) Structure of added wall.

evaluated using the Lorentz-Berthelot mixing rule. The parameters involved in the hybrid potential model are listed in Table $1 .{ }^{37}$ It is noted that the bond information, including bond stretch and angle deformation, in $\mathrm{H}_{2} \mathrm{O}$ and $\mathrm{C}_{10}$ molecules are considered by the harmonic model (see the work by Harris et $a l .^{38}$ ). Additionally, the dihedral properties of $\mathrm{C}_{10}$ molecules are also considered by the optimized potentials for liquid simulations (OPLS) model. The charges of $\mathrm{C}_{10}$ atoms are calculated by $\mathrm{DMol}^{3}$ module of Materials Studio 7.0. The potential parameters have been verified by our group, ${ }^{30,31}$ in which the densities of the water and decane bulk phase, the decane-water interfacial thickness and the decane-water interfacial tension are calculated and show good agreements with the experimental works.

In addition, different three-phase wettabilities are considered by changing the energy parameter $\varepsilon$ in eqn (1). For simplicity, we assume that the energy parameter $\varepsilon$ in the principal interactions can be written as $\mathrm{C} \cdot \varepsilon$. The constant $C$ will enable us to selectively increase or decrease the interactions between different types of atoms. For convenience, we assign the different types of interactions as SW and SO, which represent the water-solid interactions and the decane-solid interactions. Here we consider several cases as shown in Table $2 .^{39}$

Table 1 The parameters involved in the simulations. For different atoms, the hybrid potential model is adopted ${ }^{a}$

\begin{tabular}{llll}
\hline Type & $\varepsilon(\mathrm{eV})$ & $\sigma(\AA)$ & Charge $(\mathrm{e})$ \\
\hline $\mathrm{C}-\mathrm{C}$ & $2.864 \times 10^{-3}$ & 3.5000 & - \\
$\mathrm{H}^{1}-\mathrm{H}^{1}$ & $1.302 \times 10^{-3}$ & 2.5000 & - \\
$\mathrm{O}^{2}-\mathrm{O}^{2}$ & $6.611 \times 10^{-3}$ & 3.1507 & -0.834 \\
$\mathrm{H}^{2}-\mathrm{H}^{2}$ & $1.999 \times 10^{-3}$ & 0.4000 & 0.417 \\
$\mathrm{Si}-\mathrm{Si}^{3}$ & $4.033 \times 10^{-3}$ & 4.1500 & 1.1 \\
$\mathrm{O}^{3}-\mathrm{O}_{\text {bulk }}{ }^{3}$ & $2.342 \times 10^{-3}$ & 3.4700 & -0.55 \\
$\mathrm{O}^{3}-\mathrm{O}_{\text {silanol }}{ }^{3}$ & $5.290 \times 10^{-3}$ & 3.4700 & -0.675 \\
$\mathrm{H}^{3}-\mathrm{H}^{3}$ & $0.651 \times 10^{-3}$ & 1.0850 & 0.4
\end{tabular}

${ }^{a}$ Superscript: 1 represents atoms in $\mathrm{C}_{10}$ molecules, 2 represents atoms in $\mathrm{H}_{2} \mathrm{O}$ molecules, 3 represents atoms in silica.
The equilibrium contact angles in Table 2 are obtained by linear fitting of the decane-water interface points. ${ }^{34}$ By averaging the water and decane interface points calculated by the density method ${ }^{40}$ we can obtain the water-decane interface points.

\section{Results and discussion}

\section{Adsorption property}

Due to the interactions between the liquid molecules and the silica atoms, the liquid molecules can be adsorbed onto the silica surface to form a high-density zone. ${ }^{41}$ The three-phase contact line phenomenon occurs at where the liquid-vapor/ liquid interface meets the solid surface..$^{14,42-44}$ Therefore, it is very crucial to investigate the adsorption characteristics of the liquid molecules on the silica surfaces to deeply understand the moving behaviors of the molecules near the three-phase contact line. The molecular density distribution along $z$-direction is achieved by dividing the simulation regions into bins. Each $\Delta z$ $=0.5 \AA$ in size and the $z$ coordinates are stored for the $\mathrm{H}_{2} \mathrm{O}$ and $\mathrm{C}_{10}$ molecules in the bins. Then we calculate the number density distribution based on eqn (3).

Table 2 Simulation cases. $C_{\text {sw }}$ represents the energy coefficients of water-solid interactions. $C_{\text {so }}$ represents the energy coefficients of decane-solid interactions. The contact angle means the water side (displacing liquid) contact angle in the LLS system

\begin{tabular}{llll}
\hline Case & $C_{\text {SW }}$ & $C_{\text {SO }}$ & Contact angle $\left(^{\circ}\right)$ \\
\hline Case 1 & 1 & 1 & 45 \\
Case 2 & 0.8 & 1 & 50 \\
Case 3 & 0.6 & 1 & 60 \\
Case 4 & 0.4 & 1 & 65 \\
Case 5 & 0.3 & 1 & 75 \\
Case 6 & 0.2 & 1 & 90 \\
Case 7 & 0.2 & 1.1 & 95 \\
Case 8 & 0.2 & 1.2 & 110 \\
Case 9 & 0.2 & 1.5 & 120 \\
Case 10 & 0.2 & 2 & 133
\end{tabular}



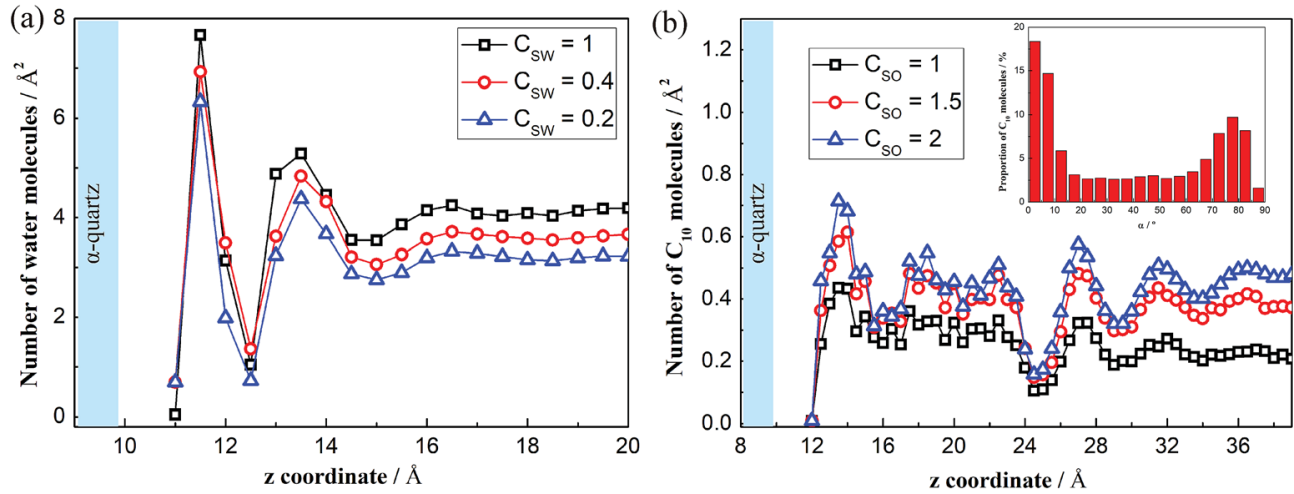

Fig. 2 Density distributions of the water and decane molecules on the solid surfaces. (a) Distribution of the water molecules. (b) Distribution of the decane molecules.

$$
D=n(b) S
$$

where $n(b)$ represents the number of elements in the $b$ th bin and $S$ is the projected area of the elements on the $x-y$ plane.

Fig. 2 shows the number density distribution along the direction perpendicular to the solid surface for $\mathrm{H}_{2} \mathrm{O}$ and $\mathrm{C}_{10}$ molecules at several cases. As seen in Fig. 2(a), the $\mathrm{H}_{2} \mathrm{O}$ molecular density distribution is non-uniform and several highdensity zones appear near the solid surfaces, meaning that the water molecules are indeed adsorbed on the solid surfaces. Away from the high-density zones, the molecular density distributes uniformly and the molecules behave like a normal liquid phase. There are three peaks at $z=11.5 \AA$, $z=13.5 \AA$ and $z=16.5 \AA$ (i.e. $0.2 \mathrm{~nm}, 0.4 \mathrm{~nm}$ and $0.7 \mathrm{~nm}$ from the O-surface), which show little difference compared to the work by Emami et $a l .{ }^{37}$ The first peak is generated due to the surface hydroxyl groups, in which zone water molecules form hydrogen bond with hydroxyls on the surface. ${ }^{41}$ The second and third peaks occur as a result of the strong interactions between the solid surfaces and the water molecules near the solid surfaces. Additionally, we see that the adsorption intensities are positively related to the interactions between the water molecules and the solid surfaces. The stronger solid-water interactions (larger $C_{\mathrm{SW}}$ ) exhibit more intense peaks, which reveal stronger adsorption forces of the solid surfaces to the liquid molecules.

For the $\mathrm{C}_{10}$ molecules, the appearing peaks demonstrate that several $\mathrm{C}_{10}$ layers are adsorbed on the solid surfaces (see Fig. 2(b)). For the bulk phase out of the layers, the population distribution oscillates weakly. We can also obtain the peaks for all the layers, where the solid surfaces have significant effects on the $\mathrm{C}_{10}$ molecules, presenting less $\mathrm{C}_{10}$ molecules adsorbed on the surfaces with weaker solid-liquid interactions (smaller $C_{\mathrm{SO}}$ ). These reflect the weaker adsorption forces of the solid surfaces to the $\mathrm{C}_{10}$ molecules. The reason of the density distribution is that $\mathrm{C}_{10}$ is a kind of chain molecule and this structure would affect the distribution of the molecules in a confined channel, especially in nanoscale channel. ${ }^{45}$ To understand more about the effects of solid surfaces on the $\mathrm{C}_{10}$ molecules, we define an angle $\alpha$ to analyze the orientation arrangement of the $\mathrm{C}_{10}$ molecules. The $\mathrm{C}$ atoms in a $\mathrm{C}_{10}$ molecule are signed from $\mathrm{C}_{1}$ to $\mathrm{C}_{10}$. For convenience and brevity, we use $\mathrm{C}_{1}, \mathrm{C}_{5}$, and $\mathrm{C}_{10}$ to determine the plane of $\mathrm{C}_{10}$. Angle $\alpha$ is obtained by averaging the angle between line $\mathrm{C}_{1} \mathrm{C}_{5}$ and silica surface and the angle between line $\mathrm{C}_{1} \mathrm{C}_{10}$ and silica surface. Owing to the strong solidliquid interactions, the $\mathrm{C}_{10}$ molecules in the near-wall region adopt a predominately parallel orientation to the surface, ${ }^{\mathbf{4 6}}$ as shown in the illustration in Fig. 2(b).

\section{Tripping and critical force}

Due to the external driving force $F$, the three-phase contact line may move during the displacing processes. However, it is found that several cases will occur with changing the driven force. When the driving force is not large enough to push the system, the three-phase contact line and the water-decane interface will be static. Oppositely, a too large driving force will cause continuously interfacial deformations of the water-decane interface, and the contact line will not reach to a steady velocity, we call this the instable case. A reasonable driving force will allow the contact line and water-decane interface to reach a stable state, in which cases the contact line can achieve a steady velocity. Fig. 3 shows the different cases with changing the three-phase wettability and the driving force $F$. Here we define the tripping force and the critical force as the minimum

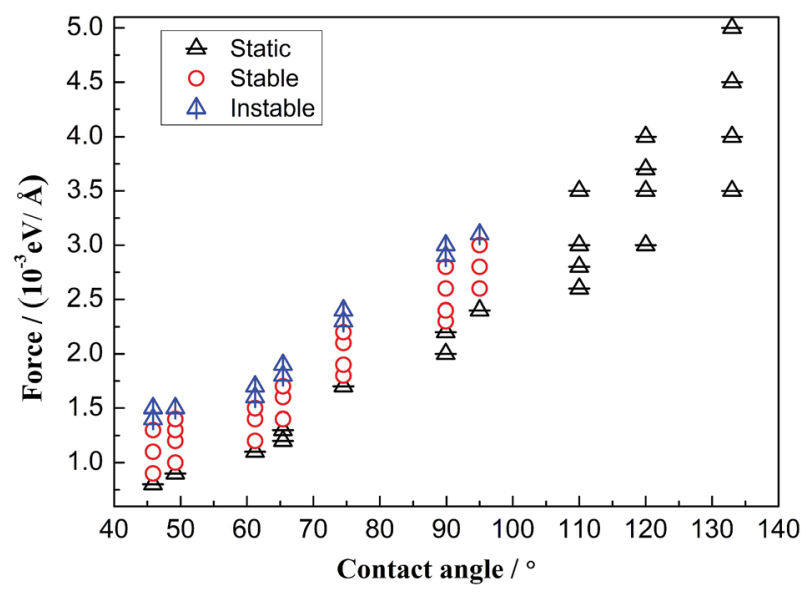

Fig. 3 Different cases at different three-phase wettabilities and driving forces. 
force to drive the contact line moving and the maximum force to keep the contact line moving stably, respectively. As we can see from Fig. 3, the tripping force and the critical force increase with decreasing the hydrophilicity. In order to better study the moving mechanism of the three-phase contact line, we analyze the stable cases under the forces between tripping force and critical force. It's worth noting that when the three-phase contact angle is larger than $110^{\circ}$, the strong decane-solid interactions will cause the approximately static case of the three-phase contact line.

\section{Moving mechanisms}

In order to further explain the moving mechanism of the threephase contact line, we obtain the molecule morphology near the contact line in the systems with different three-phase wettabilities. Several periods which reflect different moving mechanisms are given in Fig. 4(a). The contact line positions along $x$ direction are signed and one typical displaced $\mathrm{C}_{10}$ molecule is highlighted, combining with the morphology of the waterdecane interface near the solid surface. As we can see, when the three-phase contact angle is $46^{\circ}$, it seems that the $\mathrm{C}_{10}$ molecules are firstly rolled up by $\mathrm{H}_{2} \mathrm{O}$ molecules (several $\mathrm{H}_{2} \mathrm{O}$ molecules are highlighted by red color at $0 \mathrm{ps}$ ) and then run away from the solid surface. The $\mathrm{C}_{10}$ molecules repeat this process and are rolled up by the water molecules, which allow the contact line to move continuously. For this situation, after the $\mathrm{C}_{10}$ molecules are rolled up and move away from the solid surface, the interactions between the solid surface and the $\mathrm{C}_{10}$ molecules are weakened, thus it's easy for the $\mathrm{C}_{10}$ molecules to move forward with the deformation and the movement of the water-decane interface under the driving force. However, when the threephase contact angle is $90^{\circ}$, the $\mathrm{C}_{10}$ molecules seem to be pushed by the piston-like water phase to move forward since there is no obvious roll up process. Importantly, the $\mathrm{C}_{10}$ molecules near the contact line will move forward near the solid surface, but they will appear deformations and move to change their local positions within the three-phase contact line area. Besides, for a more hydrophobic case whose three-phase contact angle is $120^{\circ}$, the contact line appears to have little displacement during the whole period. It's found that the $\mathrm{C}_{10}$ molecules within the contact line area only exhibit the local deformations. Nevertheless, the water-decane interface will be more and more oblique since the decane molecules far away from the solid surface will move forward and a wedge-structure is formed at the three-phase contact zone.

As mentioned previously, the three-phase contact line phenomenon occurs where the liquid-vapor/liquid interface meets the solid surface. Hence, the movement and the deformation of the water-decane interface may have a relationship with the movement of the three-phase contact line. In order to deeply understand the moving processes of the three-phase contact line, the water-decane interfaces under different moving mechanisms are shown in Fig. 4(b). As we can see, for the "Roll up" mechanism, the deformation of the water-decane interface occurs at the same time when the three-phase contact line moves forward. When the "Piston" mechanism happens, large deformation of the water-decane interface is needed to obtain enough unbalanced Young stress, which makes the three-phase contact lime move suddenly. After this, the waterdecane interface moves forward with fluctuations under the driving force. However, for the "Shear" mechanism, the waterdecane interface moves with continuous deformation. As a result, a thin $\mathrm{C}_{10}$ film will remain on the solid surface. In conclusion, the deformations of the water-decane interfaces give us some insights that the variation trend of the contact line velocity over time is different under different moving mechanisms.

To clearly know the effects of the three-phase wettability on the different moving mechanisms, the phase diagram is given in Fig. 5. The diagram is obtained by considering a series of different three-phase wettabilities, from which we can see that the moving mechanism shows a strong dependence on the three-phase wettability. For a more hydrophilic surface, whose three-phase contact angle is less than $65^{\circ}$, the "Roll up" mechanism dominates the moving process of the three-phase contact line. In this case, the water molecules show a dominated role in the competitive adsorption near the solid surface because the water-solid interactions are stronger than the decane-solid interactions. In addition, for an intermediate wettability surface, whose three-phase contact angle is between $75^{\circ}$ and $95^{\circ}$, the "Piston" mechanism occurs, in which case the water-solid interactions and the decane-solid interactions seem to be comparable. Hence, there is no obvious competitive adsorption near the three-phase contact line. For a hydrophobic surface with the three-phase contact angle larger than $110^{\circ}$, the "Shear" mechanism plays the main role, owing to the strong decane-solid interactions.

\section{Time-averaged velocity}

To deeply understand the different moving mechanisms, the velocities of the three-phase contact line during the driving processes are analyzed. By intersecting the fitting line of the water-decane interface points and the solid surfaces, the coordinates of the three-phase contact line at different times can be obtained, from which we can further obtain the displacements of the contact line, as shown in the inset of Fig. 6. Thus, the average velocity of the three-phase contact line can be obtained. The average velocity is used because the thermal fluctuation effects of the molecules are weaker. Here we give some typical cases in different moving mechanisms. When the three-phase contact line moves in the "Roll up" mechanism, the average velocity of the three-phase contact line increases firstly and then reaches to a steady value, as shown in Fig. 6(a). In this case, the competitive adsorption of the water and the decane molecules plays an important role, together with the interfacial deformation generated by the driving force. It is noted that for a relatively large driving force $\left(F=1.3 \times 10^{-3} \mathrm{eV} \AA^{-1}\right)$, the velocity of the three-phase contact line has a peak during the accelerating period, which reflects an inertia effect under a large driving force. When the "Piston" mechanism happens, the three-phase contact line has a maximum velocity at the start-up stage, which shows a significant inertia effect. Then the velocity decreases 
(a)

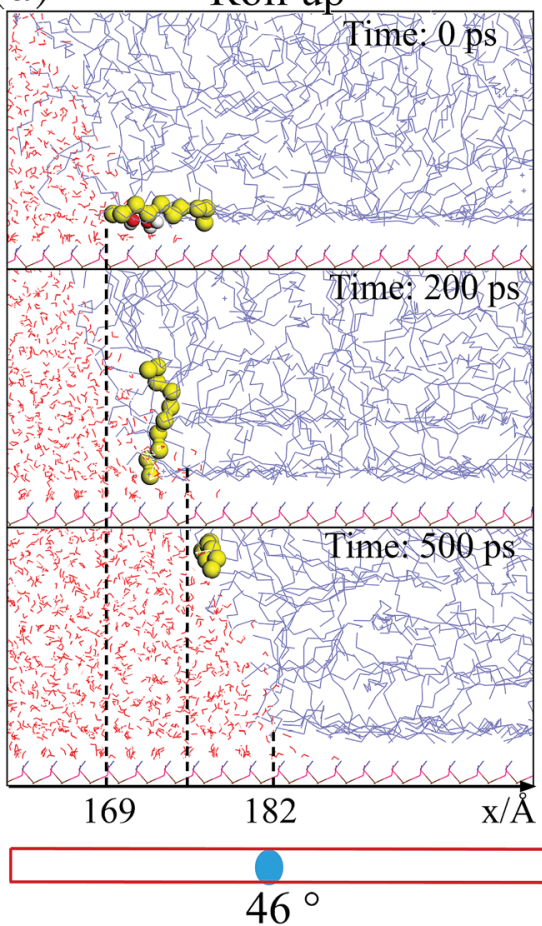

Piston

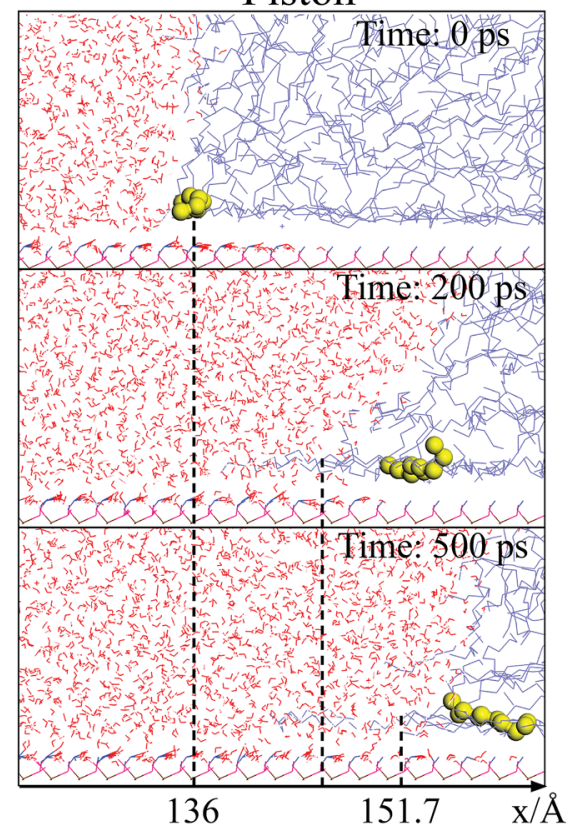

Shear

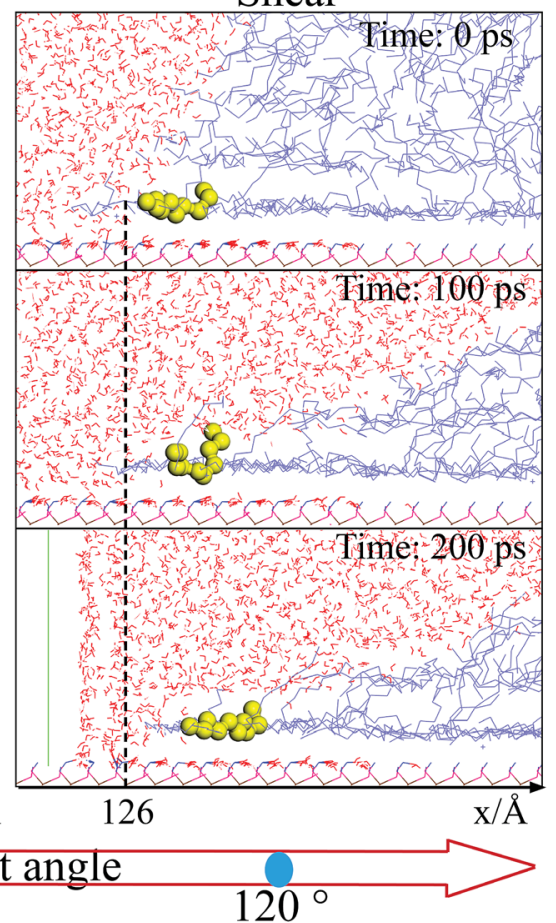

(b)
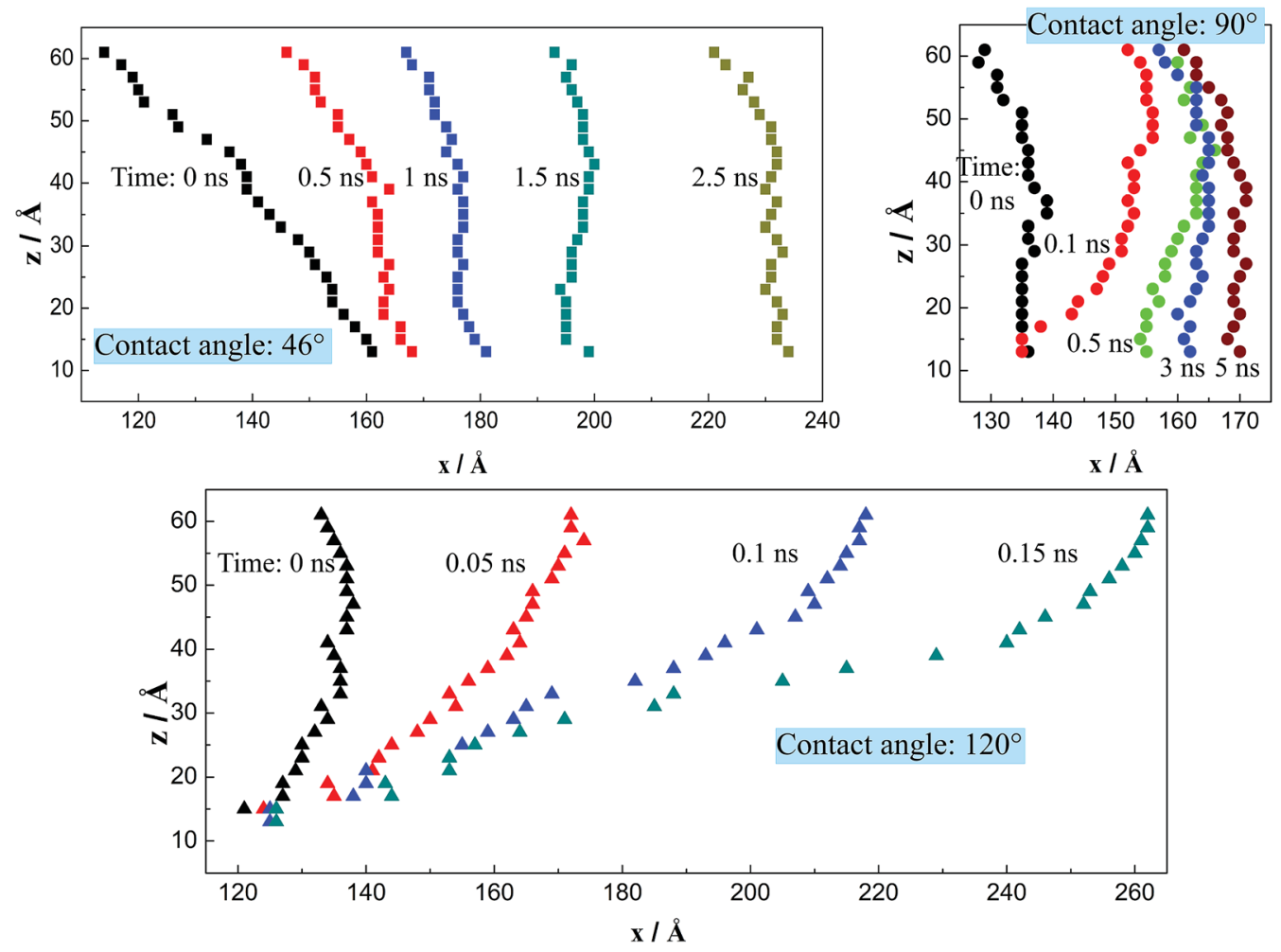

Fig. 4 Moving mechanisms of the contact line at different three-phase wettabilities. (a) Different moving modes of the displaced molecules, one typical $\mathrm{C}_{10}$ molecule is highlighted by yellow color. (b) The movement and deformation of the water-decane interfaces.

gradually and reaches to a steady value, as shown in Fig. 6(b). However, for the "Shear" mechanism, the three-phase contact line may move or jump back and forth, but will finally be zero, as shown in Fig. 6(c). This is mainly due to the strong interactions between the decane molecules and the solid surfaces.
However, it is noted that the velocity is about zero in a "Shear" case and the thermal fluctuations of the liquid molecules will cause a large error in the calculation of the contact line velocity. ${ }^{47}$ 


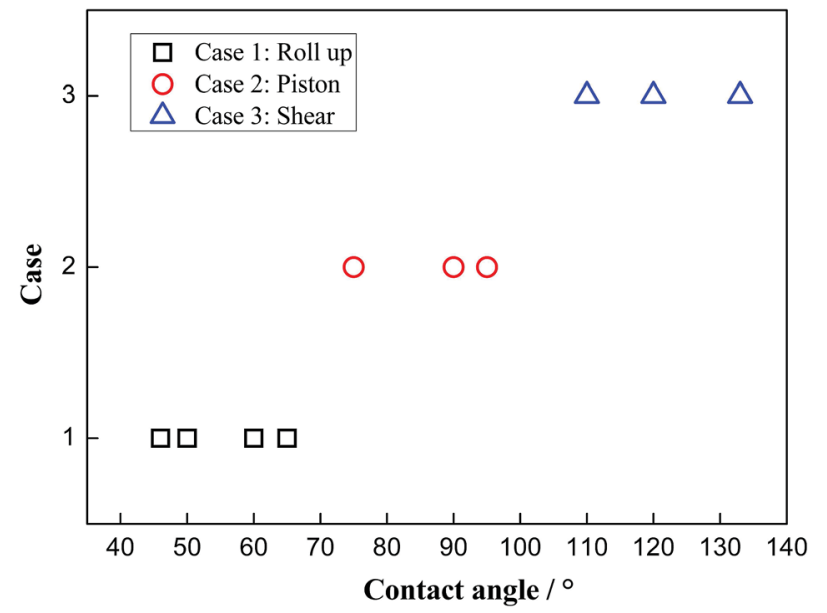

Fig. 5 Phase diagram of the moving mechanisms with the changing of three-phase wettability.

To further explain the phenomena, we analyze the driving force and the resistant force of the three-phase contact line. The driving force in our simulations is the unbalanced Young force generated by the external force. The resistant force has a relationship with the velocity of the three-phase contact line and the friction coefficient, which depends on the properties of the two liquids and the solid surfaces. ${ }^{23}$ Hence, for a given system, the velocity of the three-phase contact line is proportional to the unbalanced Young force, which can be obtained through the dynamic and the static contact angle. ${ }^{22,23}$ For the "Roll up" mechanism, the competitive adsorption between water and decane molecules makes the three-phase contact line easily move even though the unbalanced Young force is small at the initial stage. Therefore, the dynamic contact angle changes gradually with the deformation of the water-decane interface until it achieves a relatively steady state. Oppositely, the "Piston" mechanism needs a large deformation of the water-decane interface to obtain enough unbalanced Young force to drive the three-phase contact line to move. However, after the contact line starts to move, the unbalanced Young force decreases and fluctuates with the deformation of the water-decane interface, which will decrease the velocity of the three-phase contact line until it reaches to a steady value. Additionally, for the "Shear" mechanism, the strong interactions between the decane molecules and the solid surfaces cause the decane molecules hard to be driven. To generate enough unbalanced Young force, the continuous deformation of the water-decane interface happens, causing a wedgestructure in the contact line zone, and a decane film will finally remain on the surface.

\section{MKT parameters}

In order to further explain these physical phenomena from a quantitative perspective, we try to obtain the MKT parameters which reflect the physical mechanisms. The MKT derived by Blake and Haynes ${ }^{17}$ can describe the relationship between the
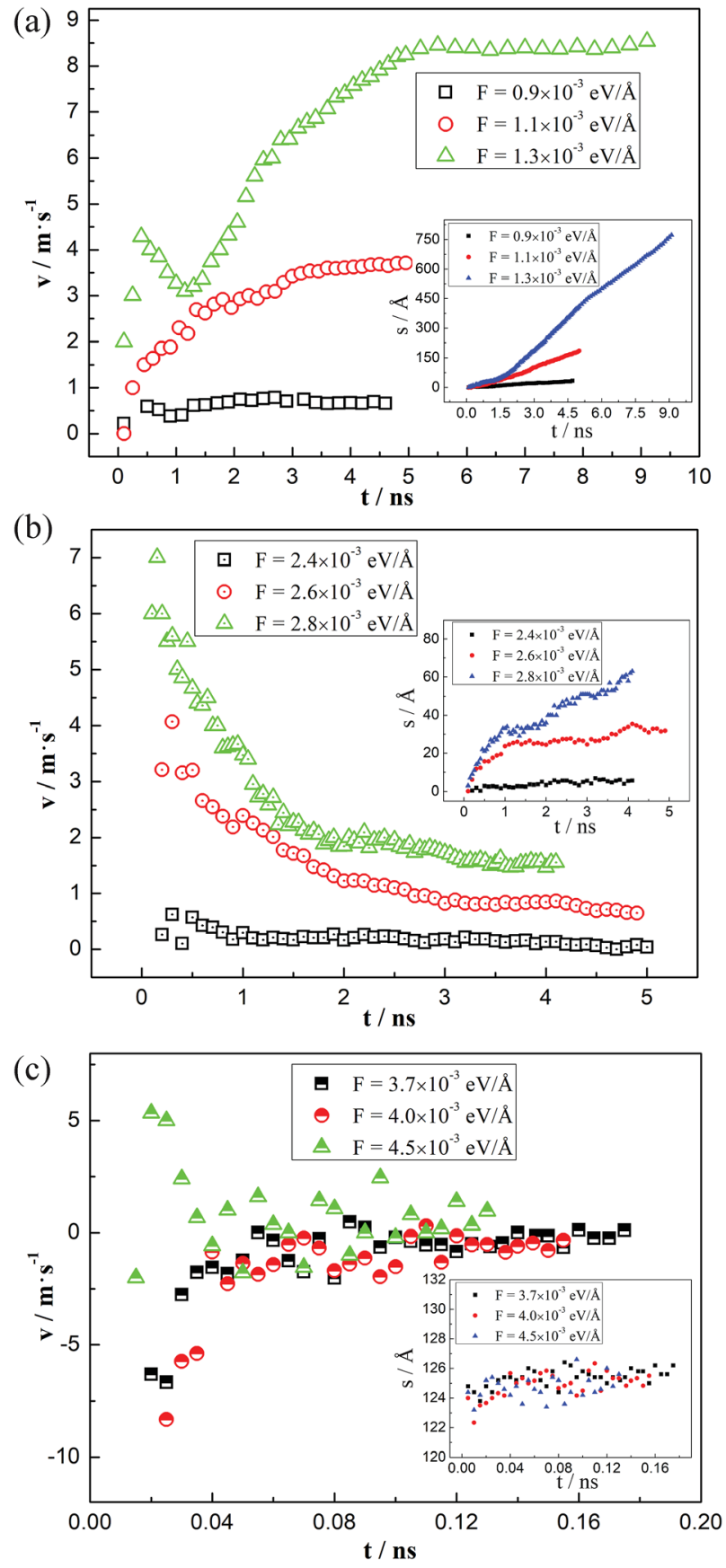

Fig. 6 Time-averaged velocity of the contact line at different displacing mechanisms. (a) Roll up $\left(46^{\circ}\right)$ : the contact line velocity increases firstly and gradually reaches a steady value. (b) Piston $\left(90^{\circ}\right)$ : the contact line velocity is maximum at the start-up stage and then decreases until reaches to a steady value. (c) Shear $\left(120^{\circ}\right)$ : large fluctuations occur but the final contact line velocity is about zero. Inseted figures show the displacements of the contact line over the simulation time.

contact line velocity $v$ and the dynamic contact angle $\theta_{\mathrm{d}},{ }^{21}$ which is given by eqn (4),

$$
v=2 K_{0} \lambda \sinh \left[\frac{\lambda^{2} \gamma_{\mathrm{L}_{1} \mathrm{~L}_{2}}}{2 k_{\mathrm{B}} T}\left(\cos \theta_{0}-\cos \theta_{\mathrm{d}}\right)\right]
$$


(a)

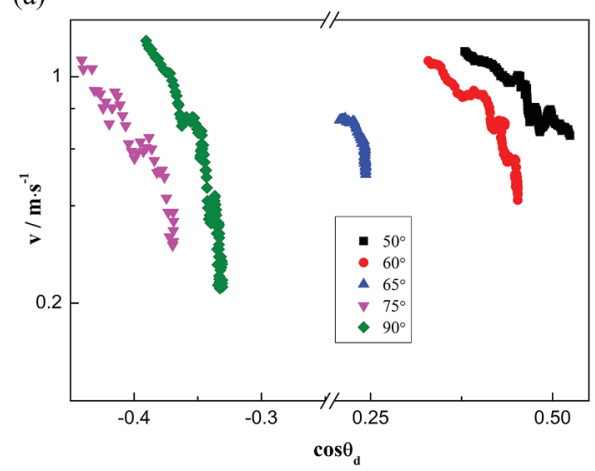

(b)

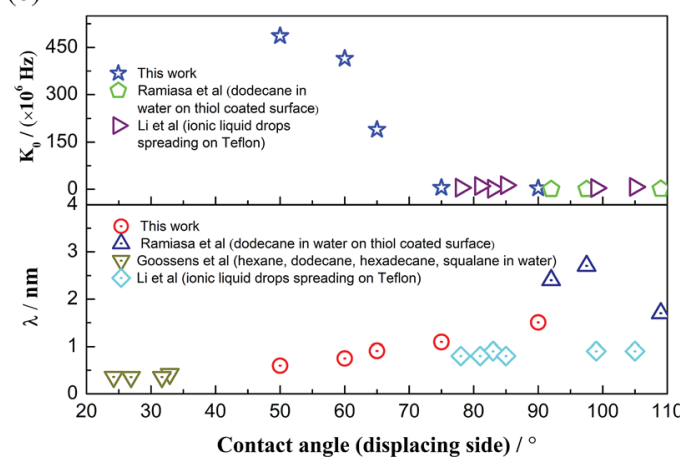

Fig. 7 MKT parameters. (a) Velocity plotted in logarithmic scale versus $\cos \theta_{\mathrm{d}}$. (b) Parameters $\lambda$ and $K_{0}$ of the MKT at different three-phase wettabilities and the comparison with other works.

where $k_{\mathrm{B}}$ and $T$ are the Boltzmann constant and the absolute temperature, respectively. $\gamma_{L_{1} L_{2}}$ is the water-decane interfacial tension (44.34 $\mathrm{mN} \mathrm{m}^{-1}$, calculated in our group ${ }^{31}$ ), and $\theta_{0}$ is the equilibrium three-phase contact angle in the displacing side. The equation also contains two molecular parameters, $K_{0}$ and $\lambda$, which are the displacement frequency and the mean distance, respectively.

In this work, a method which is used by Renate Fetzer et al. ${ }^{48}$ is adopted to obtain the two parameters. We plot the velocity $v$ in logarithmic scale versus the cosine of the instantaneous contact angle, as shown in Fig. 7(a). Since some researchers found that the MKT will predict the moving contact line well at low capillary numbers, ${ }^{18-20}$ we try to use the low velocity region to get a better adaptation of the $\mathrm{MKT}^{23} K_{0}$ and $\lambda$ are directly obtained by reducing eqn (4) to a single exponential form for large arguments and fitting the data in Fig. 7(a) with a straight line (see the work by Renate Fetzer et al. ${ }^{48}$ ), as shown in Fig. 7(b), which display good agreement with other works at the same three-phase wettability. ${ }^{21,22,49}$ The parameter $\lambda$ increases with decreasing the hydrophilicity and the value is of the same order of magnitude as other works. However, the $K_{0}$ in the "Roll up" cases are approximately 2 orders of magnitude higher than those in the "Piston" cases, which further demonstrates the different moving mechanisms from a quantitative view of points. To understand the implications of $K_{0}$, we give the usual form of $K_{0}$ by the following equation, ${ }^{22,48}$

$$
K_{0}=\frac{k_{\mathrm{B}} T}{\eta v_{L}} \exp \left[-\frac{\Delta G_{\mathrm{S}}^{*}}{N_{\mathrm{A}} k_{\mathrm{B}} T}\right]
$$

where $\eta$ and $v_{\mathrm{L}}$ are the viscosity and the volume of unit flow of the liquid phase, and $N_{\mathrm{A}}$ is the Avogadro's number. Here $\Delta G_{\mathrm{S}}^{*}$ is the activation free energy of the surface part. Usually, $\Delta g_{\mathrm{S}}^{*}=\Delta G_{\mathrm{S}}^{*} / \lambda^{2} N_{\mathrm{A}} \approx W_{\mathrm{a}}=\gamma_{\mathrm{L}_{1} \mathrm{~L}_{2}}\left(1+\cos \theta_{0}\right),{ }^{22}$ thus $K_{0}$ can be written as

$$
K_{0}=\frac{k_{\mathrm{B}} T}{\eta v_{\mathrm{L}}} \exp \left[-\frac{\lambda^{2} \gamma_{\mathrm{L}_{1} \mathrm{~L}_{2}}\left(1+\cos \theta_{0}\right)}{k_{\mathrm{B}} T}\right]
$$

$v_{\mathrm{L}}$ is the parameter which reflects the single-molecule volume for the single-molecule adsorption-desorption mechanism. In our systems, $v_{\mathrm{L}}$ is hard to be obtained since the viscosity in eqn
(6) is coupled by the two liquids and hard to know in a LLS system. However, we can speculate about the $v_{\mathrm{L}}$ depending on the different mechanisms since we can know from eqn (6) that $v_{\mathrm{L}}$ has an inversely proportional to $K_{0}$. We can know from the value of $K_{0}$ that the $v_{\mathrm{L}}$ in "Piston" mechanism is 2 orders of magnitude higher than that in "Roll up" mechanism, the ultrahigh volume of unit flow in the "Piston" mechanism further demonstrates the invalidation of the single-molecule adsorption-desorption mechanism in the water-decane-silica systems.

\section{Conclusions}

In this work, we study the moving mechanisms of the threephase contact line in the water-decane-silica systems by using the MD simulations. In order to reveal the contact line moving mechanisms, a reasonable external driving force is employed to move the contact line with a stable state. Three different wettability-related moving mechanisms are revealed, including "Roll up", "Piston" and "Shear" mechanisms. The hydrophilic surface tends to induce the "Roll up" mechanism, where the decane molecules are rolled up by the water molecules and then move forward under the driving force; the intermediate wettability surface shows the "Piston" mechanism, in which case the decane molecules are pushed by the piston-like water phases; the hydrophobic surface tends to induce the "Shear" mechanism, where the contact line is hard to be driven. For the "Roll up" mechanism, the water-solid interactions are stronger than the decane-solid interactions and thus the decane molecules are rolled up during the competitive adsorption processes. For the "Piston" mechanism, the water-solid interactions are comparable with the decanesolid interactions, and thus the decane molecules are pushed by the water molecules without obvious competitive adsorptions. For the "Shear" mechanism, the decane-solid interactions are stronger than the water-solid interactions, causing the decane molecules hard to be driven and form a wedge-structure at the three-phase contact zone. The time-averaged velocity of the contact line during the moving process shows different trends under different moving mechanisms. When the "Roll up" mechanism happens, the increasing process takes place until 
the contact line velocity reaches to a steady value. For the "Piston" mechanism, the contact line velocity has a maximum value at the start-up stage, which shows a significant inertia effect, then a following decrease of the velocity occurs until the velocity reaches to a steady value. However, the contact line velocity in a "Shear" mechanism is around zero and fluctuates significantly since the liquid molecules have strong thermal fluctuations. Furthermore, the MKT parameters also quantitatively demonstrate the different moving mechanisms. The mean distance $\lambda$ increases with the decreasing hydrophilicity and displacement frequency $K_{0}$ in the "Roll up" mechanism is 2 orders of magnitude higher than that in the "Piston" mechanism. Notably, the ultra-high volume of unit flow in the "Piston" mechanism further demonstrates the invalidation of the singlemolecule adsorption-desorption mechanism in the waterdecane-silica systems.

In summary, water-decane-silica systems are adopted to reveal the moving mechanisms of the three-phase contact line with chain molecules. Different mechanisms may cause different energy dissipation at the moving contact line, which should be further studied. Although we reveal the mechanisms by only adopting the widely-existed decane molecules, the three mechanisms definitely exist for the other kinds of chain molecules because the local deformation and orientation arrangements of different chain molecules are similar. However, the length and branches of the chain molecules may have additional effects on the movement of the contact line in some quantitative parameters, such as velocity, critical force. Finally, it should be noted that the properties of the solid surfaces are very crucial for the moving mechanisms because they determine the relative contributions of the water-solid interactions and the decane-solid interactions.

\section{Conflicts of interest}

There are no conflicts to declare.

\section{Acknowledgements}

This work was supported by the China National Funds for Distinguished Young Scientists program (No. 51425603) and project (No. 51876169).

\section{References}

1 Y. D. Shikhmurzaev, J. Fluid Mech., 1997, 334, 211-249.

2 W. K. Chan and C. Yang, J. Micromech. Microeng., 2005, 15, 1722-1728.

3 V. Kumar and J. R. Errington, J. Chem. Phys., 2013, 139, 159. 4 P. K. Mondal, U. Ghosh, A. Bandopadhyay, D. Dasgupta and S. Chakraborty, Phys. Rev. E: Stat., Nonlinear, Soft Matter Phys., 2013, 88, 023022.

5 H. Yan and S. L. Yuan, J. Phys. Chem. C, 2016, 120, 26672674.

6 P. A. Kralchevsky, K. D. Danov, V. L. Kolev, T. D. Gurkov, M. I. Temelska and G. Brenn, Ind. Eng. Chem. Res., 2014, 44, 1309-1321.
7 F. Jiménezángeles and A. Firoozabadi, J. Phys. Chem. C, 2016, 120, 11910-11917.

8 P. Randive, A. Dalal, K. C. Sahu, G. Biswas and P. P. Mukherjee, Phys. Rev. E: Stat., Nonlinear, Soft Matter Phys., 2015, 91, 053006.

9 Y. Zhang and S.-J. Park, J. Catal., 2018, 361, 238-247.

10 Y. Zhang and S. J. Park, J. Mater. Chem. A, 2018, 6, 2030420312.

11 Y. Zhang and S.-J. Park, Appl. Catal., B, 2019, 240, 92-101.

12 N. T. Chamakos, M. E. Kavousanakis, A. G. Boudouvis and A. G. Papathanasiou, Phys. Fluids, 2016, 28, 122106-122548.

13 M. Ramiasa, J. Ralston, R. Fetzer and R. Sedev, J. Phys. Chem. C, 2012, 116, 10934-10943.

14 O. V. Voinov, Fluid Dyn., 1976, 11, 714-721.

15 E. B. Dussan V, J. Fluid Mech., 1976, 77, 665-684.

16 T. D. Blake and C. J. De, Adv. Colloid Interface Sci., 2002, 96, 21-36.

17 T. D. Blake and J. M. Haynes, J. Colloid Interface Sci., 1969, 30, 421-423.

18 P. A. Kralchevsky, K. D. Danov, V. L. Kolev, T. D. Gurkov, M. I. Temelska and G. Brenn, Ind. Eng. Chem. Res., 2005, 44, 1309-1321.

19 X. Wang, A. V. Nguyen and J. D. Miller, Int. J. Miner. Process., 2006, 78, 122-130.

20 V. L. Kolev, I. I. Kochijashky, K. D. Danov, P. A. Kralchevsky, G. Broze and A. Mehreteab, J. Colloid Interface Sci., 2003, 257, 357-363.

21 S. Goossens, D. Seveno, R. Rioboo, A. Vaillant, J. Conti and J. De Coninck, Langmuir, 2011, 27, 9866-9872.

22 M. Ramiasa, J. Ralston, R. Fetzer and R. Sedev, J. Phys. Chem. C, 2011, 115, 24975-24986.

23 D. Seveno, T. D. Blake, S. Goossens and J. De Coninck, Langmuir, 2011, 27, 14958-14967.

24 T. D. Blake, J. Colloid Interface Sci., 2006, 299, 1-13.

25 R. Fetzer, M. Ramiasa and J. Ralston, Langmuir, 2009, 25, 8069-8074.

26 Z. Tang, H. Li, D. W. Hess and V. Breedveld, Cellulose, 2016, 23, 1401-1413.

27 R. G. Xu, Y. Xiang and Y. S. Leng, J. Chem. Phys., 2017, 147, 054705.

28 M. Voue, R. Rioboo, M. H. Adao, J. Conti, A. I. Bondar, D. A. Ivanov, T. D. Blake and J. De Coninck, Langmuir, 2007, 23, 4695-4699.

29 E. Bertrand, T. D. Blake and J. De Coninck, Langmuir, 2005, 21, 6628-6635.

30 B. Wen, C. Sun and B. Bai, Phys. Chem. Chem. Phys., 2018, 20, 22796-22804.

31 B. Wen, C. Sun, B. Bai, E. Y. Gatapova and O. A. Kabov, Phys. Chem. Chem. Phys., 2017, 19, 14606-14614.

32 C. Sun and B. Bai, Phys. Chem. Chem. Phys., 2017, 19, 38943902.

33 C. Sun and B. Bai, J. Phys. Chem. C, 2018, 122, 6178-6185.

34 W. Zheng, C. Sun and B. Bai, Polymers, 2017, 9, 370.

35 A. Bródka and T. W. Zerda, J. Chem. Phys., 1996, 104, 63196326.

36 S. J. Stuart, A. B. Tutein and J. A. Harrison, J. Chem. Phys., 2000, 112, 6472-6486. 
37 F. S. Emami, V. Puddu, R. J. Berry, V. Varshney, S. V. Patwardhan, C. C. Perry and H. Heinz, Chem. Mater., 2016, 26, 2647-2658.

38 J. G. Harris and K. H. Yung, J. Phys. Chem., 1995, 99, 1202112024.

39 D. Saini, Y. Zheng and D. Rao, SPE Reservoir Eval. Eng., 2009, 12, 702-712.

40 R. Raj, S. C. Maroo and E. N. Wang, Nano Lett., 2013, 13, 1509-1515.

41 D. Argyris, N. R. Tummala, A. Striolo and D. R. Cole, J. Phys. Chem. C, 2008, 112, 13587-13599.

42 J. G. Petrov and B. P. Radoev, Colloid Polym. Sci., 1981, 259, $753-760$.
43 P. G. D. Gennes, Rev. Mod. Phys., 1985, 57, 827-863.

44 F. Brochardwyart and P. G. D. Gennes, Adv. Colloid Interface Sci., 1992, 39, 1-11.

45 S. Wang, F. Javadpour and Q. Feng, Fuel, 2016, 171, 74-86. 46 S. Wang, Q. Feng, F. Javadpour, T. Xia and Z. Li, Int. J. Coal Geol., 2015, 147-148, 9-24.

47 P. Johansson and B. Hess, Phys. Rev. Fluids, 2017, 3, 1-8.

48 R. Fetzer and J. Ralston, J. Phys. Chem. C, 2009, 113, 88888894.

49 H. Li, R. Sedev and J. Ralston, Phys. Chem. Chem. Phys., 2011, 13, 3952-3959. 